\title{
Treino cognitivo em adultos maduros e idosos: impacto de estratégias segundo faixas de escolaridade
}

\author{
AlineTexxėraFabáaio- UnivesidadedeSãoPaulo SãoPaulo, Brasil \\ Thaís BentoLimaSilva - UniversidadedeSãoPaulo, SãoPaulo, Brasil \\ Prisilla Tieni Kissaki - UniversidadedeSãoPaulo, SãoPaulo Brasil \\ Marcda Guidetti Vièra - UniversidadedeSãoPaulo SãoPaulo Brasil \\ TiagoNasimento Ordanez - UniversidadedeSãoPaulo, SãoPaulo, Brasil \\ Thalita Biandi deOlivera - UniveridadedeSãoPaulo, São Paulo, Brasil \\ Flávia OgavaAramaki - UnivesidadedeSãoPaulo SãoPaulo, Brasil \\ Patŕcia Feriera Saza - UniversidadedeSãoPaulo, SãoPaulo, Brasil \\ Mônica Sanhes Yassuda1 - UniversidadedeSãoPaulo, SãoPaulo Brasil
}

\begin{abstract}
Resumo
Objetivou-se com este estudo testar a eficácia de treino cognitivo de seis sessões, baseado na apresentação e prática de estratégias de memória (categorização) e na realização de atividades que recrutam as funções executivas, oferecido a idosos. O bjetivou-se, também, identificar e comparar as estratégias mnemônicas utilizadas pelos participantes antes e após treino, segundo faixas de escolaridade. Participaram do estudo 31 idosos, divididos em Grupo 1 (com até 8 anos de escolaridade) e Grupo 2 (com 9 anos ou mais). Foram aplicadas questões sociodemográficas, escalas cognitivas e uma lista de estratégias possíveis para identificação das estratégias usadas. Os resultados indicaram a influência da escolaridade no uso de estratégias de memória no pré-teste. No pós-teste, apontaram para aumento na velocidade de processamento e na utilização de estratégias. Concluiu-se que 0 uso de estratégias, a autoeficácia para a memória e 0 ganho após o treino cognitivo podem ser influenciados pela escolaridade.
\end{abstract}

Palavas dhave Cognição; Memória; Idosos.

Cognitive training in older adults and the elderly: impact of educational strategies acconding to age

\begin{abstract}
The objective of this study was to test the effectiveness of a cognitive training program of six sessions based on the presentation and practice of memory strategies (categorization) and activities that recruit executive functions offered for older adults. We also aimed at identifying and comparing the mnemonic strategies used by participants stratified by levels of schooling before and after training. The study included 31 older adults divided into group 1 (up to 8 years of schooling) and group 2 (9 or more years of schooling). The instruments used were: sociodemographic questionnaire, cognitive scales and a list of possible strategies to identify the strategies used. The results were indicated the influence of education on the use of memory strategies in the pre-test and post-test. At the post-test, there was a significant increase in processing speed and strategies use. It is concluded that he use of strategies, memory self-efficacy and the gain after cognitive training may be influenced by education.
\end{abstract}

Keyuards Cognition; Memory; Elderly.

\section{Entrenamiento cognitivo en adultos maduros y ancianos: impacto de estrategias según niveles de escolaridad}

\begin{abstract}
Resumen
El objetivo de este estudio fue testar la eficacia de entrenamiento cognitivo de seis sesiones basado en la presentación y práctica de estrategias de memoria (categorización) y en la realización de actividades que reclutan las funciones ejecutivas, ofrecido a ancianos. Se objetivó también identificar y comparar las estrategias mnemónicas utilizadas por los participantes antes y después del entrenamiento, según niveles de escolaridad. Participaron del estudio 31 ancianos, divididos en Grupo 1 (con hasta 8 años de escolaridad) y Grupo 2 (con 9 años o más). Fueron realizadas cuestiones sociodemográficas y utilizadas escalas cognitivas y una lista de estrategias posibles para identificación de las estrategias usadas. Los resultados indicaron la influencia de la escolaridad en el uso de estrategias de memoria en el pre test. En el post teste, señalaron aumento en la velocidad de procesamiento y en la utilización de estrategias. Se concluyó que el uso de estrategias, la autoeficacia para la memoria y el gaño después del entrenamiento cognitivo pueden ser influenciados por la escolaridad. Palabrasdave Cognición; Memoria; Ancianos.
\end{abstract}

Com 0 processo de envelhecimento, alguns subsistemas de memória sofrem alterações significativas. A memória episódica e a memória operacional estão entre os subsistemas mais alterados com o processo de envelhecimento normal (Gazzaniga \& Heatherton, 2005). Parente, O liveira e Jaeger (2006) documentaram que os déficits de memória em indivíduos idosos estão associados com dificuldades na codificação e na recuperação das informações. De acondo com Lezak, Howieson e Loring (2004), apontam que os idosos têm uma diminuição em seus recursos cognitivos (atenção, memória operacional e velocidade de processamento), e, assim, podem ter maior dificuldade de lançar mão de estratégias cognitivas para apoiar a codificação e recuperação da informação. Nesse cenário, as estratégias cognitivas são 
concebidas como um meio eficaz de compensar os déficits de memória, em particular, na memória episódica (Carvalho, Neri \& Yassuda, 2010).

As estratégias de memória podem auxiliar na organização dos materiais a serem codificados e servir como mediadores para o processamento eficiente das informações (Klingberg, 2010). As estratégias podem ser ferramentas para estimular os níveis residuais de plasticidade associados ao processo de envelhecimento (Baltes, 1997; Klingberg, 2010; Schaie, Willis \& Caskie, 2004). Encontra-se documentado que ganhos objetivos no desempenho de memória são observados após participação em programas que estimulam 0 aprendizado e prática com estratégias de memória (Ball \& cols., 2002; Schacter, 2003; West, Welch \& Yassuda, 2000; Willis, Tenndtedt, Marsiske \& Ball, 2006).

Dunlosky e Hertzog (1998) indicam que as estratégias internas, isto é, realizadas mentalmente, incluem a criação de imagens mentais, as estratégias de categorização, associação verbal, método dos lugares (mthod of loi) e método face-nome. As estratégias externas compreendem o uso de calendários, agendas, listas de afazeres, alarmes, entre outros recursos.

Entre as estratégias mais estudadas na literatura encontram-se as imagens mentais e a categorização. A criação de imagens mentais consiste na criação consciente de imagens que auxiliem no fortalecimento do traço de memória. Nessa técnica, cria-se uma imagem de uma situação ou de um item a ser memorizado e a imagem pode ajudar a dar vivacidade às informações, podendo servir de mediador no momento do resgate da informação. Isto é, ao tentar resgatar o item memorizado, a imagem pode facilitar 0 acesso à informação desejada (Y esavage, Sheik, Friedman \& Tanke, 1990).

A eficácia do uso de imagens mentais interativas é descrita em estudos de treino de memória (Lima-Silva \& cols., 2010; West, 1995). Entretanto, a criação de imagens mentais exige recursos cognitivos sofisticados e, por vezes, pode não ser uma estratégia prática para a vida diária, pois sua criação requer certa concentração e tempo. Autores defendem que a criação consciente de imagens mentais leva a um processamento mais profundo e detalhado da informação que poderia facilitar a memorização de longo prazo (Salmazo-Silva \& Yassuda, 2009).

A categorização refere-se à organização de informações em categorias lógicas ou em grupos semânticos que podem servir como pistas no momento do resgate da informação, visto que, dentro de uma categoria, os itens podem associar-se uns aos outros, fortalecendo o traço de memória. Lasca (2003) e Carvalho e cols. (2010) demonstraram a eficácia da técnica de categorização para a memorização de listas de supermercado e de figuras bidimensionais. Yassuda, Tavares, Fortes e Neri (2006) demonstraram que os idosos que receberam treino de memória passaram a usar com maior frequência a categorização para a memorização de listas. Lima-Silva e cols. (2011), em treino de oito sessões com ênfase nas estratégias de categorização, detectaram alterações no resgate da lista de palavras do CERAD.

Há algumas décadas, os pesquisadores discutem quais fatores são determinantes para 0 desempenho cognitivo na velhice (Ball \& cols., 2002). Dentre os fatores investigados, as crenças e os afetos sobre a memória recebem atenção significativa. A autoeficácia para a memória, isto é, o grau de confiança de um indivíduo sobre sua capacidade de realizar tarefas de memória tem recebido atenção significativa nos estudos sobre o envelhecimento cognitivo (West \& cols., 2000).

A autoeficácia pode, em parte, auxiliar na explicação dos déficits de memória de pessoas idosas. Diversos autores (Berry \& West, 1989; Cavanaugh, 1996; Welch \& West, 1995; Woo, SchmitterEdgecombe \& Fancher, 2008) documentaram que os idosos são mais vulneráveis à autoeficácia rebaixada, pois vivenciam dificuldades cognitivas com maior frequência e são fortemente afetados pelos estereótipos negativos sobre 0 envelhecimento. Os déficits cognitivos dos idosos são mais notados e podem ser interpretados como sinais de um declínio irreversível (Petersen, 2011). Esse processo pode levar à insegurança, que, por sua vez, pode afetar o desempenho cognitivo (Metternich, Shmidtke \& Hull, 2009; West \& cols., 2000; Yassuda, Lasca \& Neri, 2005). É plausível também que idosos com senso de autoeficácia rebaixada evitem tarefas que envolvam memorização, com implicações negativas em seu cotidiano. Evidências sugerem que a autoeficácia para memória entre idosos é significativamente mais baixa que entre adultos jovens e que a autoeficácia tende a se correlacionar positivamente com 0 desempenho objetivo em tarefas de memorização (Berry, West \& Dennehey, 1989; West \& Berry, 1989, Yassuda \& cols., 2005).

Nesse contexto, objetivou-se no presente estudo descrever as estratégias mnemônicas utilizadas espontaneamente pelos participantes, estratificados por faixas de escolaridade, antes e após o treino cognitivo. 0 treino teve como foco o ensino do uso da estratégia de categorização, a estimulação da atenção, da memória episódica e das funções executivas. Adicionalmente, objetivou-se avaliar a possibilidade de alteração de crenças negativas sobre o envelhecimento cognitivo. Até 0 momento, não existem estudos brasileiros 
avaliando o uso de estratégias em diferentes faixas de escolaridade.

\section{Participantes}

\section{Método}

Foram recrutados 31 idosos, que foram prétestados e a seguir participaram de seis sessões de treino, seguidas de pós-teste. Os participantes do treino eram adultos maduros e idosos que fizeram inscrições para a oficina D esafiando a Memória, oferecida para os participantes da Universidade Aberta à Terceira Idade da Escola de Artes, Ciências e Humanidades da Universidade de São Paulo.

Os critérios de inclusão foram: ter acima de 50 anos, ter condições sensoriais (audição e visão) suficientes para participar de atividades em grupo usando lápis e papel, ter condições mínimas de locomoção motora, não ter diagnóstico prévio de demência e depressão.

Nas análises estatísticas, os participantes foram separados em um grupo de menor escolaridade, entre 1 e 8 anos de escolaridade (Grupo 1, n=13), e um grupo de maior escolaridade, entre 9 e 16 anos (Grupo 2, $\mathrm{n}=18$ ). Para este estudo não foi recrutado um grupo controle, visto que o objetivo foi avaliar as alterações no uso de estratégias, no pré e pós-teste, em duas faixas distintas de escolaridade.

\section{Instrumentos}

Os instrumentos utilizados foram: questionário sociodemográfico, incluindo questões sobre idade, escolaridade e renda; Escala de D epressão Geriátrica com 15 questões - EDG (Almeida \& Almeida, 1999), Questionário de Autoeficácia para Memória adaptado (Berry \& cols., 1998; Yassuda \& cols., 2005), Teste de Memória Episódica de 18 Figuras (Carvalho \& cols., 2010), prova Códigos da bateria WAIS-III (Nascimento, 2002), o Teste do Desenho do Relógio (TDR) (Aprahamian, Martinelli, Neri \& Yassuda, 2010), pergunta aberta sobre 0 uso de estratégias " 0 que você fez para recordar-se das figuras?" e, logo após, uma lista contendo estratégias possiveis, para que o participante escolhesse as estratégias utilizadas. Para responder a pergunta aberta, os participantes tinham que escrever em uma folha em branco, logo após a pergunta descrita anteriormente, quais estratégias teriam utilizado.

Para avaliar a presença de sintomas depressivos, foi aplicada a EDG que é um dos instrumentos mais utilizados para o rastreamento de depressão no idoso. No Brasil, Almeida e Almeida (1999) e Paradela, Lourenço e Veras, (2005) aplicaram a EDG-15 em 64 idosos de um ambulatório psiquiátrico e ela mostrou-se confiável e válida para 0 rastreamento da depressão na população idosa brasileira, com seis pontos como ponto de corte ideal.

No Questionário de Autoeficácia para Memória, na versão adaptada, o participante é solicitado a marcar o número de itens que imagina ser capaz de memorizar, em contextos específicos de memorização, como a memorização de nomes, listas de supermercado, entre outras tarefas, ao invés de marcar seu grau de confiança no seu desempenho em diversos graus de dificuldade, como é proposto no questionário original (Yassuda \& cols., 2005). Destaca-se que a versão adaptada desse instrumento necessita de estudos sobre suas caraterísticas psicométricas.

Para avaliação da memória episódica foi utilizado o Teste de 18 Figuras, nas versões A e B. Essas figuras podem ser divididas em três categorias semânticas com seis itens cada uma. As figuras foram extraídas de um estudo brasileiro, desenvolvido por Pompéia, Miranda e Bueno (2001), que forneceram dados normativos para 0 uso de 400 figuras avaliadas por estudantes universitários e crianças de 5 a 7 anos. As pranchas A e B foram distribuídas alternadamente entre os participantes durante a sessão de avaliação. Os participantes tiveram um minuto para estudar a prancha e em seguida foram solicitados a virar a folha e completar a prova Códigos. Posteriormente, foram solicitados a escrever 0 nome das figuras que lembravam.

A prova Códigos trata-se de um subteste da bateria WAIS-III adaptada e validada para uso no Brasil (Nascimento, 2002; Nascimento \& Figueiredo, 2002). Para o presente estudo a prova códigos foi utilizada no protocolo entre a memorização das figuras e 0 resgate subsequente, para que 0 intervalo entre 0 momento de memorização e 0 resgate fosse aproximadamente de cinco minutos. Esse subteste também foi usado para avaliar a velocidade de processamento. A variável de interesse foi a quantidade de símbolos transferidos em 120 segundos, pois testagens piloto indicaram que esse intervalo de tempo evitaria o efeito teto e solo no resgate das figuras memorizadas.

No Brasil, poucos estudos investigaram o uso do TDR como instrumento de avaliação cognitiva entre idosos (Aprahamian \& cols., 2010; Fuzikawa, LimaCosta, Uchôa \& Shulman, 2007; Lourenço, RibeiroFilho, Moreira, Paradela \& Miranda, 2009; Okamoto, 2001). A prahamian e cols. (2010) documentaram que 0 TDR apresenta boa sensibilidade e especificidade para a detecção da doença de Alzheimer e boa validade convergente com o Mini Exame do Estado Mental (Brucki \& cols., 2003).

Além da pergunta aberta e da lista para investigar 0 uso de estratégias, a categorização também foi 
avaliada diretamente no protocolo de resgate das 18 figuras no pré e no pós-teste, por meio da medida de Bousfield (1953), utilizada em estudos anteriores (Carvalho \& cols., 2010; Mello, 2003). Para a obtenção desse índice, calcula-se a taxa de repetição intracategórica (ratio of repeition), seguindo a fórmula $[R R=r / n-1]$, onde $r$ representa 0 número de itens de uma mesma categoria recordados juntos e $\mathrm{n} 0$ total de itens recordados da lista. Esse índice varia de 0 (nenhuma categorização) a 1 (categorização total).

\section{Procedimentos}

O treino cognitivo ocorreu uma vez por semana e cada sessão tinha duração de duas horas. Os participantes realizavam atividades em conjunto (grande grupo) por uma hora, coordenados por um docente, e depois eram divididos em grupos menores (pequeno grupo), coordenados por graduandos do curso de gerontologia, na segunda hora.

No grande grupo, os idosos receberam informações sobre os diferentes tipos de memória (explícita e implícita, de curta duração e de longa duração), sobre as estratégias mnemônicas, crenças sobre memória, e envelhecimento cerebral normal e patológico. Os participantes aprenderam sobre as estratégias internas de memória, como, por exemplo, associação, criação de imagens mentais e categorização, e também sobre o uso de estratégias externas, como agenda, calendário, caderno e organização do ambiente. Além disso, solicitavam esclarecimentos de dúvidas sobre os conteúdos e relatavam se haviam usado alguma estratégia aprendida nas últimas aulas. Os participantes também foram encorajados a relatar momentos nos quais tinham conseguido apresentar bom desempenho de memória.

No pequeno grupo, os idosos realizavam atividades que estimulavam 0 uso das estratégias mnemônicas. A estratégia treinada com maior frequência foi a de categorização. Foram utilizadas embalagens de supermercado que eram categorizadas de acordo com sua finalidade. As embalagens continham preço para que os participantes pudessem calcular mentalmente 0 valor de uma compra simulada. Com moedas e notas de brinquedo realizaram tarefas de pagamento e troco. A cada encontro os idosos tinham que lembrar-se de realizar uma tarefa de casa (ver descrição dos encontros em Anexo). A programação das atividades do treino seguiu protocolo semelhante de estudos anteriores (Lima-Silva \& cols., 2011), e as sessões estão descritas em anexo.

Nas análises estatísticas, a comparação das variáveis categóricas entre os grupos de escolaridade foi feita por meio do teste quiquadrado, ou o teste exato de Fisher (na presença de valores esperados menores que 5). Faixa de escolaridade (baixa $\mathrm{x}$ alta) e tempo da avaliação (pré $\mathrm{x}$ pós) foram consideradas variáveis independentes. As variáveis cognitivas e de uso de estratégias foram consideradas as variáveis dependentes. Em virtude da ausência de distribuição normal, o teste U de Mann-Whitney foi usado para comparar as diferenças entre as faixas de escolaridade e o teste de Wilcoxon, para examinar os dados de evolução do pré para o pós-teste. Adicionalmente, foram calculados deltas (escore do pós-teste menos 0 escore do pré-teste para cada variável cognitiva) para examinar os ganhos após o treino. Para analisar a relação entre as variáveis numéricas foi utilizado 0 coeficiente de correlação de Spearman. O nível de significância adotado para os testes estatísticos foi de $5 \%$, ou seja, $p<0,05$. Os dados foram digitados no Programa Epidata versão 3.1. Para análise estatística foi utilizado o programa computacional SPSS v.17.0.

\section{Resultados}

Na Tabela 1, observa-se que os participantes do Grupo 1 e do Grupo 2 apresentaram diferenças estatisticamente significativas quanto à escolaridade e renda. $\mathrm{O}$ Grupo 2 apresentava indivíduos mais escolarizados e com maior renda.

Na Tabela 2, observa-se que no pré-teste e no pós-teste os indivíduos do Grupo 1 apresentaram mais sintomas depressivos. Para a autoeficácia, assim como para as variáveis cognitivas e para a medida de Bousfield, os participantes do Grupo 2 apresentaram desempenho mais elevado no pré-teste e maior ganho entre 0 pré e 0 pós teste. Não foram encontradas alterações no TDR em função de efeito teto documentado no pré-teste. 
Tabela 1. Perfil sociodemográfico dos participantes $(\mathrm{n}=31)$

\begin{tabular}{|c|c|c|c|c|c|c|}
\hline \multirow{3}{*}{ Variável } & & \multicolumn{4}{|c|}{ Grupos } & \multirow{3}{*}{$\mathrm{p}$} \\
\hline & & \multicolumn{2}{|c|}{ Grupo $1(\mathrm{n}=13)$} & \multicolumn{2}{|c|}{ Grupo $2(\mathrm{r}=18)$} & \\
\hline & & Frequência & Porcentagem & Frequência & Porcentagem & \\
\hline \multirow[t]{2}{*}{ Sexo } & Masculino & 0 & 0,00 & 5 & 27,78 & \multirow[t]{2}{*}{$0,058^{\mathrm{a}}$} \\
\hline & Feminino & 13 & 100,00 & 13 & 72,22 & \\
\hline \multirow{3}{*}{$\begin{array}{l}\text { Rendimento } \\
\text { familiar }\end{array}$} & A té dois salários mínimos & 3 & 23,08 & 2 & 11,11 & \multirow[t]{3}{*}{$0,015^{\mathrm{b}}$} \\
\hline & Mais de 2 a 4 salários mínimos & 9 & 69,23 & 6 & 33,33 & \\
\hline & Mais de 4 salários mínimos & 1 & 7,69 & 10 & 55,56 & \\
\hline \multirow[t]{3}{*}{ Trabalha } & Não & 9 & 69,23 & 13 & 72,22 & \multirow[t]{2}{*}{$1,000^{a}$} \\
\hline & Sim & 4 & 30,77 & 5 & 27,78 & \\
\hline & & \multicolumn{2}{|c|}{ Grupo $1(\mathrm{n}=13)$} & \multicolumn{2}{|c|}{ Grupo $2(\mathrm{n}=18)$} & $\mathrm{F}$ \\
\hline \multirow{5}{*}{$\begin{array}{l}\text { Idade } \\
\text { (53 a } 81 \text { anos) }\end{array}$} & Mínimo & \multicolumn{2}{|c|}{56,00} & \multicolumn{2}{|c|}{53,00} & $0,733^{b}$ \\
\hline & Máximo & \multicolumn{2}{|c|}{74,00} & \multicolumn{2}{|c|}{81,00} & \\
\hline & Mediana & \multicolumn{2}{|c|}{65,00} & \multicolumn{2}{|c|}{64,50} & \\
\hline & q1 & \multicolumn{2}{|c|}{62,00} & \multicolumn{2}{|c|}{59,00} & \\
\hline & q3 & \multicolumn{2}{|c|}{70,00} & \multicolumn{2}{|c|}{68,00} & \\
\hline Escolaridade & Mínimo & \multicolumn{2}{|c|}{1,00} & \multicolumn{2}{|c|}{9,00} & $<0,001^{b}$ \\
\hline \multirow[t]{4}{*}{ (1 a 16 anos) } & Máximo & \multicolumn{2}{|c|}{8,00} & \multicolumn{2}{|c|}{$\begin{array}{l}16,00 \\
1100\end{array}$} & \\
\hline & Mediana & \multicolumn{2}{|c|}{4,00} & & 1,00 & \\
\hline & q1 & \multirow{2}{*}{\multicolumn{2}{|c|}{$\begin{array}{l}3,00 \\
8,00\end{array}$}} & & \\
\hline & q3 & & & \multicolumn{2}{|c|}{14,00} & \\
\hline
\end{tabular}

a. Teste Exato de Fisher; b. Teste U de Mann-Whitney; Grupo 1 ( $\mathrm{n}=13)=1$ a 8 anos de escolaridade; Grupo 2 (n=18)= 9 a 16 anos de escolaridade.

Tabela 2. Mediana e intervalos interquartílicos entre parênteses para as variáveis dependentes no pré e pós-teste

\begin{tabular}{|c|c|c|c|c|c|c|c|c|}
\hline \multirow{2}{*}{ Variáveis } & \multirow{2}{*}{ Grupo } & \multicolumn{3}{|c|}{ Grupo 1} & \multicolumn{3}{|c|}{ Grupo 2} & \multirow{2}{*}{$\mathrm{p}^{*}$} \\
\hline & & Mediana & Q1 & Q3 & Mediana & Q1 & Q3 & \\
\hline \multirow[t]{2}{*}{ EDG } & Pré-teste & 3,00 & 2,50 & 5,50 & 2,00 & 1,00 & 3,50 & 0,046 \\
\hline & Pós-teste & 4,00 & 2,00 & 5,00 & 2,00 & 1,00 & 3,00 & 0,045 \\
\hline \multirow{4}{*}{$\begin{array}{l}\text { Autoeficácia itens de } \\
\text { supermercado } \\
\text { Autoeficácia fotos }\end{array}$} & Pré-teste & 10,00 & 6,00 & 10,00 & 14,00 & 10,00 & 14,00 & 0,034 \\
\hline & Pós-teste & 10,00 & 10,00 & 14,00 & 10,00 & 10,00 & 14,00 & 0,661 \\
\hline & Pré-teste & 4,00 & 2,00 & 6,00 & 4,00 & 4,00 & 6,00 & 0,368 \\
\hline & Pós-teste & 4,00 & 2,00 & 4,00 & 6,00 & $6,00^{a}$ & $6,00^{\mathrm{a}}$ & 0,002 \\
\hline \multirow{4}{*}{$\begin{array}{l}\text { Autoeficácia objetos } \\
\text { guardados } \\
\text { Códigos a }\end{array}$} & Pré-teste & 10,00 & 6,00 & 10,00 & 10,00 & 10,00 & 14,00 & 0,076 \\
\hline & Pós-teste & 10,00 & 6,00 & 10,00 & 14,00 & $10,00^{a}$ & $14,00^{a}$ & 0,016 \\
\hline & Pré-teste & 29,00 & 21,00 & 33,00 & 45,50 & 33,00 & 56,00 & 0,007 \\
\hline & Pós-teste & 28,00 & 22,00 & 46,00 & 57,50 & $45,00^{b}$ & $68,0^{b}$ & 0,004 \\
\hline \multirow[t]{2}{*}{18 Figuras } & Pré-teste & 10,00 & 9,00 & 11,00 & 11,00 & 10,00 & 13,00 & 0,084 \\
\hline & Pós-teste & 11,00 & 8,00 & 14,00 & 15,50 & 14,00 a & $17,00^{a}$ & 0,006 \\
\hline \multirow[t]{2}{*}{ Medida de Bousfield } & Pré-teste & 0,42 & 0,33 & 0,60 & 0,56 & 0,40 & 0,75 & 0,081 \\
\hline & Pós-teste & 0,56 & $0,40^{\mathrm{a}}$ & $0,85^{\mathrm{a}}$ & 0,81 & $0,71^{b}$ & $0,86^{b}$ & 0,074 \\
\hline \multirow[t]{2}{*}{ TDR } & Pré-teste & 5,00 & 3,00 & 5,00 & 5,00 & 4,00 & 5,00 & 0,189 \\
\hline & Pós-teste & 5,00 & 1,00 & 5,00 & 5,00 & 4,00 & 5,00 & 0,246 \\
\hline
\end{tabular}

Notas: Escala de Depressão G eriátrica (EDG), Itens extraídos do Questionário de Autoeficácia: recordação de lista de compras de 18 itens (18 itens), fotos de 10 pessoas e identificação de nomes (fotos), identificação de 18 objetos colocados em locais diferentes (18 objetos), Teste do D esenho do Relógio (TDR). * Teste U de Mann-Whitney, $\mathrm{p}<0,05$ a. Para esta variável neste grupo houve diferença entre o pré e pós-teste no teste de Wilcoxon $\mathrm{p}<0,05$. b. Para esta variável neste grupo houve diferença entre o pré e pós-teste no teste de Wilcoxon p<0,001. 
Tabela 3. Estratégias relatadas espontaneamente na pergunta aberta sobre o uso de estratégias para memorizar as figuras, na amostra estratificada por escolaridade

\begin{tabular}{lcccc}
\hline \multirow{2}{*}{ Variável } & \multicolumn{2}{c}{ Pré-teste (r) } & \multicolumn{2}{c}{ Pós-teste (n) } \\
\cline { 2 - 5 } & Grupo 1 & Grupo 2 & Grupo 1 & Grupo 2 \\
\hline Categorização* & 1 & 6 & 5 & 15 \\
Memorização & 10 & 7 & 4 & 1 \\
Associação & 0 & 3 & 2 & 1 \\
Imagem & 0 & 1 & 0 & 1 \\
Repetição & 0 & 1 & 0 & 0 \\
A través da Primeira Letra & 0 & 2 & 0 & 1 \\
Decorou a O rdem & 0 & 1 & 0 & 1 \\
Contou o total das figuras & 0 & 1 & 0 & 0 \\
Não usou nenhuma estratégia & 2 & 1 & 2 & 1 \\
Total de Emissões** & 11 & 22 & 11 & 20 \\
\hline
\end{tabular}

${ }^{*}$ Categorização (Pós-teste): Grupo 1 f Grupo 2; Teste Exato de Fisher com p<0,05

**Total de emissões (Pré-teste): G rupo $1 \neq$ G rupo 2; Teste U de Mann-Whitney com $\mathrm{p}<0,05$

Tabela 4. Avaliação do uso de estratégias por meio da lista de estratégias, para memorizar as figuras, na amostra estratificada por escolaridade

\begin{tabular}{lcccc}
\hline \multirow{2}{*}{ Variável } & \multicolumn{2}{c}{ Pré-teste (n) } & \multicolumn{2}{c}{ Pós-teste (r.) } \\
\cline { 2 - 5 } & Grupo 1 & Grupo 2 & Grupo 1 & Grupo 2 \\
\hline Criou uma imagem mental das figuras & 9 & 14 & 12 & 14 \\
Organizou as figuras em grupos para se lembrar* & 2 & 11 & 9 & 16 \\
Fez alguma associação para se recordar das figuras & 5 & 7 & 11 & 12 \\
Usou as primeiras letras das figuras para se recordar & 4 & 5 & 3 & 1 \\
Pensou em objetos que possui na bolsa ou em casa & 2 & 3 & 7 & 4 \\
Contou quantas figuras havia no total de cada categoria & 1 & 4 & 3 & 9 \\
Pensou na ordem que as figuras apareceram & 2 & 6 & 5 & 3 \\
Usou outra estratégia para se lembrar das figuras & 1 & 3 & 4 & 7 \\
Total de estratégias marcadas & 26 & 53 & 54 & 66 \\
\hline
\end{tabular}

${ }^{*}$ Organizou as figuras em grupos para se lembrar (Pré-teste): G rupo $1 \neq$ G rupo 2; Teste quiquadrado com $\mathrm{p}<0,05$

Por meio da Tabela 3, observa-se que no préteste, na pergunta aberta sobre uso de estratégias, a maioria dos participantes do Grupo 1 relatou fazer uso da memorização, isto é, relataram tentar memorizar as figuras. O Grupo 2 relatou usar uma variedade de estratégias. Entretanto, no pós-teste, 0 Grupo 2 relatou usar exclusivamente a estratégia de categorização, enquanto o Grupo 1 relatou o uso de diversas estratégias. Na contagem geral, o Grupo 2 relatou maior uso de estratégias nos dois momentos de avaliação.

A Tabela 4 revela, por meio dos resultados da pergunta fechada (lista de estratégias), que os $\mathrm{G}$ rupos 1 e 2 escolheram diversas estratégias no pré e pósteste, e não houve diferença entre os grupos nas escolhas realizadas. No pós-teste, após a intervenção, houve aumento no número de estratégias em ambos os grupos (Tabela 4).

\section{Discussão}

Os estudos realizados anteriormente (Verhaeghen \& Salthouse, 1997; Willis \& cols., 2006; West, 1995) sugeriram que o treino cognitivo pode promover mudanças no funcionamento cognitivo em idosos e colaborar para a funcionalidade e independência. Os estudos de treino cognitivo ainda são escassos no Brasil, não havendo dados suficientes para confirmar se os achados em populações internacionais podem ser generalizados para a nossa população em virtude das características sociodemográficas diferentes. Nesse contexto, objetivou-se no presente estudo investigar o impacto do treino no uso de estratégias de memória entre adultos maduros e idosos, separados em duas faixas de escolaridade, e identificar e comparar as estratégias mais utilizadas espontaneamente e após intervenção cognitiva oferecida aos grupos.

Adicionalmente, comparou-se o desempenho em tarefas de memória episódica, velocidade de 
processamento e autoeficácia entre os grupos divididos em indivíduos com menor escolaridade (Grupo 1) e indivíduos com maior escolaridade (Grupo 2).

As estratégias mais usadas no pré-teste foram a memorização (relato de esforço cognitivo) e a criação de imagens mentais em ambos os grupos. No pósteste, houve maior utilização das estratégias de imagens mentais e categorização, o que sugere que os indivíduos do estudo passaram a utilizar as estratégias ensinadas nos treinos, demonstrando aprendizagem. Foi observado que tanto o Grupo 1 quanto o Grupo 2 passaram a utilizar a estratégia de categorização com maior frequência após a intervenção. Esse dado está em congruência com o estudo de Carvalho e cols. (2010), que encontrou que houve diferença significativa na intensidade da categorização entre 0 pré e o pós-teste para o grupo experimental.

A autoeficácia dos participantes do grupo menos escolarizado (Grupo 1) foi mais baixa que a relatada pelos indivíduos mais escolarizados (G rupo 2). Entretanto, parece não ter ocorrido interação entre a autoeficácia e o impacto decorrente do treino, visto que os dois grupos apresentaram melhora quanto ao uso das estratégias no pós-teste.

West, Bagwell e Dark-Freudeman (2007) encontraram dados que divergem dos apresentados no presente estudo. Os autores realizaram estudo de treino cognitivo multifatorial com 0 intuito de melhorar a autoeficácia e o desempenho dos idosos em tarefas de memória. Oitenta e quatro participantes receberam treino para memorizar listas de supermercado, nomes e estórias. Os resultados mostraram que houve melhora significativa quanto à autoeficácia no grupo experimental, que também utilizou as estratégias de memorização de modo mais eficaz quando comparados ao grupo controle. Esses dados ressaltam a importância de novos estudos sobre metamemória e autoeficácia, visto que estudos sugerem que a melhora na autoeficácia pode interagir com o desempenho cognitivo de idosos após treino.

No presente estudo foi possível perceber que apesar da melhora após treino e 0 impacto do treino para os grupos de ambas as escolaridades, o grupo com maior escolaridade e maior autoconfiança (Grupo 2) apresentou melhores resultados.

Os participantes do Grupo 2 apresentaram maior velocidade de processamento antes e após a intervenção. Esses achados estão alinhados a estudos anteriores, que ressaltaram que indivíduos com maior escolaridade apresentam maior nível de reserva cognitiva, que pode ser entendida como processamento mais eficiente (Stern, 2002). Outras pesquisas, como a de Aarsten, Smits, Van Tiburg,
Knipscheer e Deeg (2003) e Wilson, Cockburn, Baddeley e Hiorns (1989), salientaram que a escolaridade pode estar associada a ambientes e a estilos de vida diferentes que também podem modular o desempenho cognitivo na velhice.

Entre as características da intervenção que podem estar associadas aos resultados positivos do treino estão o uso de tarefas semelhantes às tarefas do cotidiano, o grau ascendente de complexidade das tarefas propostas e 0 trabalho em pequenos grupos com graduandos. Adicionalmente, nos grupos, os participantes eram pareados por nível de escolaridade, 0 que os deixava menos ansiosos diante do próprio desempenho nas tarefas propostas. Essas características parecem facilitar o desenvolvimento de treino cognitivo para pessoas idosas.

Entre as limitações do estudo, menciona-se que este não incluiu um grupo controle. 0 estudo ofereceu um número limitado de sessões, realizadas em uma frequência semanal. Outros protocolos devem testar a eficácia de treinos mais longos e com menor espaço temporal entre as sessões. Adicionalmente, não houve avaliação dos ganhos associados ao treino na vida cotidiana. $O$ fato dos participantes terem escrito as estratégias que utilizaram pode ter influenciado os dados provenientes dos participantes menos escolarizados. Por fim, a amostra foi relativamente pequena e composta de idosos ativos. D essa forma, os achados podem não ser representativos de outros grupos de idosos.

\section{Referências}

Aarsten, M. J., Smits, C. H., Van Tiburg, T., Knipscheer, K. C. P., \& Deeg, J. H. (2003). Activity in older adults: cause or consequence of cognitive functionning? A longitudinal study on everyday activities and cognitive performance in older adults, The Jaumals of Geoutdogy, 57 B(2), 153-162.

Almeida, O ., \& Almeida, S. A. (1999). Reability of the Brasilian version of the Geriatric Scale (GDS) short form. Arquivos de Neurqpiquiatria, 57(2B), 421-426.

Aprahamian, I., Martinelli, J. E., Neri, A. L., \& Yassuda, M. S. (2010). Clock D rawing Test accuracy compared to standard screening tests in Alzheimers disease: results from a study in a sample of Brazilian elderly with heterogeneous educational background. Intemational Psychogeiatrics, 22, 64-71.

Ball, K., Berch, D. B., Helmers, K. F., Jobe, L. B., Leveck, M. D., Marsiske, M., Morris, J. N., 
Rebok, G. W., Smith, D. M., Tennstedt, S. L., Unverzagt, F. W., \& Willis, S. L. (2002). Effects of cognitive training interventions with older adults. JAMA, 288(18), 2271-2281.

Baltes, P. B. (1997). On the incompletude architecture of human ontogeny: selection, optimization and compensation as founding of developmental theory. Amaican Psychdogist, 52, 366-380.

Berry, J. M., West, R. L., \& Dennehey, D. M. (1989). Reliability and validity of the self-efficacy questionnaire. Deddqmetal Psydhogy, 25, 701713.

Berry, J. M., West, R. L., \& D ennehey, D. M. (1998). Reliability and validity of the memory selfefficacy questionnaire. Dexdqpmental Psychdogy, 25, 701-713.

Bousfield, W. A. (1953). The occurrence of clustering in the recall of randomly arranged associates. Jaumal of Geneal Psydhdogy, 49, 229-240.

Brucki, S. M. D., Nitrini, R., Caramelli, P., Bertolucci, P. H. F., \& Okamoto, I. H. (2003) Sugestões para o uso do mini-exame do estado mental no Brasil. Arquivos de Nerropiquiatria, 61(3-B), 777781.

Carvalho, F. C. R., Neri, A. L., \& Yassuda, M. S. (2010). Treino de memória episódica com ênfase em categorização para idosos sem demência e depressão. Psicogàa Rellexão eCńtica, 23(2), 317323.

Cavanaugh, J. C. (1996). Memory self-efficacy as a moderator of memory change. Em: $\mathrm{F}$. Blanchard-Fields \& T. M. Hess (O rgs.), Pespetives on cogitive hange in adulthood and aging (pp. 488-507). New Y ork: MacG raw-Hill.

Dunlosky, J., \& Hertzog, C. (1998). Aging and deficits in associative memory: what is the role of strategy production? Psydhdogy and Aging 13(4), 597-607.

Fuzikawa, C., Lima-Costa, M. F., Uchôa, E., \& Shulman, K. (2007). Correlation and agreement between the Mini-mental State Examination and the Clock D rawing Test in older adults with low levels of schooling: the Bambuí Health Aging Study (BHAS). Intemational Psychogeiatric, 19(4), 657-67.

Gazzaniga, M. S., \& Heatherton, T. F. (2005). Ciênia psicóógca: mente céłdro e compatameto Porto Alegre: Artmed.
Klingberg, T. (2010). Training and plasticity of working memory. Trends in Cognitive Säenes, 14, 317-324.

Lasca, V. B. (2003). Trénamento de memónia no endheimeto nomal: um programa aplicado a idbsos (Dissertação de Mestrado). Universidade Estadual de Campinas, Campinas, São Paulo, Brasil.

Lezak, M. D., Howieson, D. B. \& Loring, D. W. (2004). Neuropsydogical assesment. Nova Iorque: Oxford University Press, 2004.

Lima-Silva, T. B., Ordonez, T. N., Dias-Santos, G., Fabrício, A. T., Aramaki, F. O., Almeida, E. B., Vianna-Paulo, D. L., Malagutti, M. P., ValenteOliveira, A. C., Iwasaki, A., Souza, G. S., \& Yassuda, M. S (2010). Effects of cognitive training based on metamemory and mental images. Dementia \& Neuropshyodoga, 4(2), 114119.

Lima-Silva, T. B. L., Valente-Oliveira, A. C., Malagutti, M. P., Vianna-Paulo, D. L., Danzini, V., \& Yassuda, M. S. (2011). Treino cognitivo para idosos baseado em estratégias de categorização e cálculos semelhantes a tarefas do cotidiano. Reista Brasilera de Geiatria e Gerontdoga, 14, 65-74.

Lourenço, R. A., Ribeiro-Filho, S. T., Moreira, I. F. H., Paradela, E. M. P., \& Miranda, A. S. (2009). The Clockdraw-ingtest: performance among elderly with low educational level. Reista Brasilera dePsiquiatria, 30(4), 309-315.

Mello, C. B. (2003). Estratééjas cateegricas dereecrdação e formação deconcitos emcianças destea quartozeanos de idade (Tese de Doutorado). Universidade de São Paulo, São Paulo, Brasil.

Metternich, B., Shmidtke, K., \& Hull, M. (2009). How are memory complaints in functional memory disorder related to measures of affect, metamemory and cognition? Jaumal of PsychoscmaticRerch 66(5), 435-44.

Nascimento, E. (2002). Adaptação e validação do teste WAIS-III para contexto brasilero (Tese de Doutorado). Universidade de Brasília, Brasília, Brasil.

Nascimento, E., \& Figueiredo, V. L. M. (2002). WISC-III e WAIS-III: alterações nas versões originais americanas decorrentes das adaptações para uso no Brasil. Psicdoga: Rellexão e Cútica, 15(3), 603-612. 
O kamoto, I. H. (2001). Aspetos cogitivos da doença de alzhimer no teste do réóga avaliação de amostra da população brasileira (Tese de D outorado). Universidade Federal de São Paulo, São Paulo, Brasil.

Paradela, E. M. P., Lourenço, R. A., \& Veras, R. (2005). Validação da escala de depressão geriátrica em um ambulatório geral. Revista de SaúdePública, 39(6), 918-923.

Parente, M. M. P., Oliveira, A. A., \& Jaeger, A. (2006). Cogrição e endheimento São Paulo: Artmed.

Petersen, M. D. (2011). Mild cognitive impairment. The New Engand Jaumal of Medione, 364(23), 2227-2234.

Pompéia, S., Miranda, M. C., \& Bueno, O. L. A. (2001). A set of 400 pictures standardised for protuguese. Arquivos de Nemrpsiquiatria, 59(2-B), 330-337.

Salmazo-Silva, H., \& Yassuda, M. S. (2009). Memory training for older adults with low education: mental images versus categorization. Educational Geontdogy, 35, 890-905.

Schacter, D . L. (2003). Os stepecados da memóna: coma mentelembra esquee São Paulo: Artmed.

Schaie, K. W., Willis, S. L., \& Caskie, G. I. L. (2004). The Seattle longitudinal study: relation between personality and cognition. Aging Nerropsydhogy and Cognition, 11, 304-234.

Stern, Y. (2002). What is cognitive reserve? Theory and research application of the reserve concept. Jaumal of the Intemational Neurqpsydhdogical Socity, 8, 448-460.

Verhaeghen, P., \& Salthouse, T. A. (1997). Metaanalysis of age-cognition relation in adulthood: estimates of linear and nonlinear age effects and structural models. Psychdogial Bullein, 122(3), 231-249.

West, R. L. (1995). Compensatory strategies for ageassociated memory impairment. Em A. D. Baddeley, B. A. Wilson \& F. N. Watts (Ed.), Handbook of Memory Disardes (pp. 481-500). John Wiley e Sons Ltda.

West, R. L., Bagwell, D. K., \& Dark-Freudeman, A. (2007). Self-Efficacy and memory aging: the impact of a memory intervention based on selfefficacy. Aging Nemrpsychdogyand Cogition, 15(3), 1-28.
West, R. L., \& Berry, J. M. (1994). Age declines in memory self-efficacy: general or limited to particular tasks and measures? Em: J. D. Sinnott (O rg.), Handbodk of adult lifespan leaming (pp. 426446). New Y ork: Greenwood Press.

Welch, D. W., \& West, R. W. (1995). Self-efficacy and mastery: its applications to issues of environmental control, cognition and aging. Dexdopmental Rexiev 15, 150-171.

West, R. L., Welch, D. C., \& Yassuda, M. S. (2000). Innovative approaches to memory training for older adults. Em R. Hill, L. Backman \& A. S. Neely (O rg.), Cognitive rehabilitation in old age (pp. 81-105). Nova Iorque, Oxford: Oxford University Press.

Willis, S. L., Tenndtedt, M., Marsiske, M., \& Ball, K. (2006). For the ACTIVE study group long-term effects of cognitive trainning on everyday functional outcomes in older adults. JAMA, 296(23), 2805-2814.

Wilson, B., Cockburn, J., Baddeley, A., \& Hiorns, R. (1989). The development and validation of a test battery for detecting and monitoring everyday memory problems. Jaumal Clinical and Expeimental Neuropsychdogy, 11(6), 855- 70.

Woo, E., Schmitter-Edgecombe, M., \& Fancher, J. B. (2008). Memory prediction accuracy in younger and older adults: a cross-sectional and longitudinal analysis. Aging Neuropsyddogy, and Cognition, 15, 68-94.

Yassuda, M. S., Lasca, V. B., \& Neri, A. L. (2005). Meta-memória e auto-eficácia: um estudo de validação de instrumentos de pesquisa sobre memória e envelhecimento. Psiclogia: Reflexão e Cútica, 18(1), 78-90.

Yassuda, M. S., Tavares, S. S., Fortes, A. G., \& Neri, A. L. (2006). Treino de memória em idosos saudáveis: Mecanismos e benefícios. Psicogja. RellexãoeCńtica, 19, 470-481.

Y esavage, J. A., Sheik, J. I., Friedman, L., \& Tanke, E. (1990). Learning mnemonics: roles of aging and subtle cognitive impairment. Psydhogy and Aging 5(1), 133-137.

Recdidoem05/ 04/ 2011 Reformladbem01/ 03/ 2012 Aprovadoem25/ 03/ 2012 
Anexo 1- Descrição das atividades de treino cognitivo

\section{Sessão 1}

No grande grupo: Aula introdutória sobre envelhecimento cognitivo; estruturas cerebrais importantes para a memória; subsistemas da memória; introdução ao uso de estratégias mnemônicas.

Nos pequenos grupos: tarefa de categorização com 20 itens de supermercado; tarefa de atenção e funções executivas do livro D eu Branco, onde sequências de 10 palavras eram lidas e os participantes deveriam repeti-las em ordem inversa (p. 89); resgate dos itens de supermencado.

Tarefa para casa: trazer uma receita preferida.

\section{Sessão 2}

No grande grupo: Revisão da aula anterior e introdução de novos conceitos de neurociências, como descrição de estruturas cerebrais e habilidades cognitivas que são recrutadas durante a realização de tarefas de memória.

Nos pequenos grupos: Foi realizada a tarefa de categorização em grupo com 25 itens de supermercado. Em seguida, foi feita uma tarefa de interferência para treino de funções executivas e atenção: exercício do livro D eu Branco, onde sequências horizontais eram lidas e depois os participantes deveriam colocá-las na ordem em que elas ocorressem (p. 92). D epois dessa atividade, foi feito o resgate dos itens de supermercado, pedindo para que os participantes escrevessem os itens que se recordassem. Tarefa de casa: os participantes precisavam trazer uma fotografia e relatar alguma lembrança associada a ela.

\section{Sessão 3}

No grande grupo: Foram apresentadas as estratégias mnemônicas principais, como a criação de imagens mentais, associações visuais e verbais, memorização e categorização.

Nos pequenos grupos: A tarefa de casa da fotografia foi transformada em tarefa de memória, perguntando quem trouxe cada uma delas (após socialização). Foi realizada tarefa de categorização em grupo com 25 itens de supermercado (com um novo conjunto de itens). A instrutora realizou com todos os participantes o agrupamento dos itens, depois os retirou e os colocou em uma sacola. Em seguida, houve uma tarefa de interferência para treino de funções executivas e atenção: exercício do livro Deu Branco, onde os participantes deveriam ler sequências horizontais e dizer as palavras na ordem inversa (p. 89) com 3 e 4 itens (p. 90). Foi feito o resgate dos itens de supermercado, pedindo para os participantes escreverem os itens que se recordassem. Tarefa de casa: foi pedido aos participantes que trouxessem uma piada para contar ao grupo.

\section{Sessão 4}

No grande grupo: Breve revisão sobre a aula anterior e aula sobre a importância da categorização, dando exemplos e exercícios a serem realizados em sala.

Nos pequenos grupos: Foi retomada a tarefa de casa (piadas). Em seguida, foi feita uma prática com soma mental e tarefa de troco. Na primeira rodada, os idosos pegaram três itens com preços etiquetados (embalagens do minimercado), somaram mentalmente e pagaram com o dinheiro exato usando notas e moedas. Na segunda rodada, os idosos pegaram três itens, somaram mentalmente e pagaram apenas com notas (deveriam pagar com 0 valor maior, receber e conferir mentalmente o troco recebido em moedas). Em seguida, foi perguntado quais foram os itens que cada idoso comprou na primeira rodada. Tarefa de casa: os participantes deveriam trazer a letra de uma música preferida.

\section{Sessão 5}

No grande grupo: Conversa sobre as crenças de memória e os sucessos da memória com os idosos.

Nos pequenos grupos: Foi feita a socialização das músicas preferidas. Em seguida, trabalhou-se com embalagens do mini-mercado. Os idosos selecionaram quatro itens, pagaram somente com notas, mas receberam obrigatoriamente o troco errado. Os participantes tinham que conferir o troco mentalmente e dizer qual era o valor correto. Foi realizada a atividade STOP, trabalhando fluência verbal com restrição categórica e fonológica. Os participantes tiveram que pensar em itens como frutas, animais, nomes próprios e de lugares (cidade, estado ou país) iniciando com uma determinada letra. Foram resgatados os itens comprados. Tarefa de casa: foi pedido aos participantes que contassem no próximo encontro uma travessura do passado. 


\section{Sessão 6}

No grande grupo: Foi realizada uma avaliação para verificar a percepção dos idosos em relação à oficina de memória. Aula introdutória e de fechamento mostrando como estruturas cerebrais podem ser moduladas pelo estilo de vida ativo e estimulação cognitiva.

Nos pequenos grupos: Foi feita a socialização das travessuras escolhidas. Em seguida, houve a simulação de tarefa de compra e troco com 4 ou 5 itens. Nesse dia, um participante foi o comprador e outro fez o papel do caixa, fornecendo o troco. Foi pedido aos participantes que memorizassem os itens que cobraram do colega. D epois, foi realizada a tarefa do livro Deu Branco, organizar ideias para formar frases. Foi feito o resgate dos itens do supermercado que foram memorizados.

Sobre os autores:

Aline Teixeira-Fabnício é bacharel em G erontologia da Escola de Artes, Ciências e Humanidades da Universidade de São Paulo.

Thaís Bento Lima-Silva é bacharel em Gerontologia da Escola de Artes, Ciências e Humanidades da Universidade de São Paulo. Mestranda em Neurologia pela Faculdade de Medicina da Universidade de São Paulo.

Priscilla Tiemi Kissaki é bacharel em Gerontologia da Escola de Artes, Ciências e Humanidades da Universidade de São Paulo.

Marcela Guidetti Vieira é graduanda em Gerontologia da Escola de Artes, Ciências e Humanidades da Universidade de São Paulo.

Tiago Nascimento Ordonez é bacharel em Gerontologia da Escola de Artes, Ciências e Humanidades da Universidade de São Paulo.

Thalita Bianchi de Oliveira é bacharel em Gerontologia da Escola de Artes, Ciências e Humanidades da Universidade de São Paulo.

Flávia Ogava Aramaki é bacharel em Gerontologia da Escola de Artes, Ciências e Humanidades da Universidade de São Paulo.

Patrícia Ferreira Souza é bacharel em Gerontologia da Escola de Artes, Ciências e Humanidades da Universidade de São Paulo.

Mônica Sanches Yassuda é Professora Associada de Psicogerontologia da Escola de Artes, Ciências e Humanidades da Universidade de São Paulo.

Contato com os autores:

Escola de Artes Ciências e Humanidades, US. Av. Arlindo Bettio, 1000; Ermelino Matarazzo - SP 03828-000

Email: yassuda@usp.br 